\title{
AN ACCURATE HEADING SOLUTION USING MEMS-BASED GYROSCOPE AND MAGNETOMETER INTEGRATED SYSTEM (PRELIMINARY RESULTS)
}

\author{
M. El-Diasty ${ }^{\mathrm{a}, \mathrm{b}}$ \\ ${ }^{a}$ Hydrographic Surveying Department, Faculty of Maritime Studies, King Abdulaziz University (mkandeel@kau.edu.sa) \\ ${ }^{\mathrm{b}}$ Engineering Department of Public Works, Faculty of Engineering, Mansoura University
}

Technical Commission II

KEY WORDS: Gyroscope, Magnetometer, Heading, Integrated Solution, MEMS, Low Cost

\begin{abstract}
:
An accurate heading solution is required for many applications and it can be achieved by high grade (high cost) gyroscopes (gyros) which may not be suitable for such applications. Micro-Electro Mechanical Systems-based (MEMS) is an emerging technology, which has the potential of providing heading solution using a low cost MEMS-based gyro. However, MEMS-gyro-based heading solution drifts significantly over time. The heading solution can also be estimated using MEMS-based magnetometer by measuring the horizontal components of the Earth magnetic field. The MEMS-magnetometer-based heading solution does not drift over time, but are contaminated by high level of noise and may be disturbed by the presence of magnetic field sources such as metal objects. This paper proposed an accurate heading estimation procedure based on the integration of MEMS-based gyro and magnetometer measurements that correct gyro and magnetometer measurements where gyro angular rates of changes are estimated using magnetometer measurements and then integrated with the measured gyro angular rates of changes with a robust filter to estimate the heading. The proposed integration solution is implemented using two data sets; one was conducted in static mode without magnetic disturbances and the second was conducted in kinematic mode with magnetic disturbances. The results showed that the proposed integrated heading solution provides accurate, smoothed and undisturbed solution when compared with magnetometerbased and gyro-based heading solutions.
\end{abstract}

\section{INTRODUCTION}

An accurate heading estimation is required for many applications such as urban, indoor, pedestrian and marine navigation when GPS signals are denied. High grade gyroscope (gyro) can provide accurate heading that meets the navigation requirements for such applications but with very high cost which may not suitable for such applications. Micro-Electro Mechanical Systems-based (MEMS) is an emerging technology, which has the potential of providing heading solution using a low cost MEMS-based gyro. Unfortunately, however, MEMS-based gyro measurements are contaminated by high level of noise along with biases and the heading solution drifts significantly over time (El-Diasty and Pagiatakis, 2008; Titterton, 2004).

On the other hand, MEMS-based magnetometers have the potential of providing magnetic heading and this estimate is obtained by measuring the horizontal components of the Earth magnetic field. Magnetometers suffer from the same type of gyros and accelerometers errors; however, their impact is smaller since magnetometers measurements are not integrated to form the final heading estimate. Thus main error impacting magnetometers is the disturbances of the magnetic field due to the presence of iron and other magnetic materials in the near environment of the magnetometers. This type of error is very complicated to model (because unpredictable). Under certain conditions, this error can be calibrated using the swinging method, however, it is ineffective when the environment changes. Therefore, magnetic disturbances must be considered when accurate heading estimation is required (Ali, et al., 2012; Tian et al., 2014).

Heading estimation was investigated by many researchers based on GPS-only heading, Gyro-only based heading, magnetometer-based heading and traditional integration methods (Kubrak, 2007; Godha et al., 2006; Afzal et al., 2011). However, accurate heading estimation using low cost MEMS-based gyros and magnetometers integration is currently under investigation, particularly for indoor navigation. This paper proposed an accurate heading estimation procedure based on the integration of gyro and magnetometer measurements that can correct gyro and magnetometer measurements. In the proposed procedure, the gyro angular rates of changes are estimated (derived) from magnetometer measurements. Then, the estimated and measured gyro angular rates of changes are integrated with a robust filter to estimate the heading.

To test the performance of the estimated heading, two data sets were implemented by the proposed integrated method. The first data set was conducted in static mode and without magnetic disturbances. The second data set was conducted in kinematic mode with magnetic disturbances. The comparison was made between the proposed accurate heading estimation procedure, magnetometer-based and gyro-based heading estimation 


\section{METHODOLOGY}

The methodology can be classified into two steps:

Step 1: Estimation of angular rates of changes from magnetometer measurements

Step 2: Development of an accurate integrated heading solution

\subsection{Estimation of Angular Rate of Changes from Magnetometer Measurements}

The Earth magnetic field varies very slowly in time and space and the slow change is negligible in low dynamic navigation applications. Therefore, changes in the magnetic field expressed in the coordinate system of the platform that measured by magnetometers are only due to changes in the orientation of the platform relative to the inertial frame. The above hypothesis leads to following theoretical relationship that connects gyro and magnetometer measurements:

$$
\begin{aligned}
& \omega_{x}^{e s t}=\frac{d \varphi_{m}}{d t}=\frac{d\left(\arctan \left(m_{y}^{m e s} / m_{z}^{m e s}\right)\right)}{d t} \\
& \omega_{y}^{e s t}=\frac{d \theta_{m}}{d t}=\frac{d\left(\arctan \left(m_{x}^{\text {mes }} / m_{z}^{m e s}\right)\right)}{d t} \\
& \omega_{z}^{e s t}=\frac{d \psi_{m}}{d t}=\frac{\left.d \arctan \left(\begin{array}{c}
m_{y}^{m e s t} / m_{x}^{m e s} \\
d t
\end{array}\right)\right)}{d t}
\end{aligned}
$$

where

$\varphi_{m}, \theta_{m}, \psi_{m}$ are the magnetic roll, pitch and heading parameters,

$\omega_{x}^{e s t}, \omega_{y}^{e s t}$ and $\omega_{z}^{e s t}$ are the three estimated angular rate of changes,

$m_{x}^{m e s}, \quad m_{y}^{m e s}$ and $m_{z}^{m e s}$ are the three

magnetometer measurements.

Equations 1, 2 and 3 are simplified and provide the estimated angular rate of changes using magnetometer measurements and magnetometer derivatives as follows:

$$
\begin{aligned}
& \omega_{x}^{e s t}=\frac{\dot{m}_{y}^{e s t} \cdot m_{z}^{m e s}-m_{y}^{m e s} \cdot \dot{m}_{z}^{e s t}}{\left(m_{y}^{m e s}\right)^{2}+\left(m_{z}^{m e s}\right)^{2}} \\
& \omega_{y}^{e s t}=\frac{\dot{m}_{x}^{e s t} \cdot m_{z}^{m e s}-m_{x}^{m e s} \cdot \dot{m}_{z}^{e s t}}{\left(m_{x}^{m e s}\right)^{2}+\left(m_{z}^{m e s}\right)^{2}}
\end{aligned}
$$

$$
\omega_{z}^{e s t}=\frac{\dot{m}_{y}^{e s t} \cdot m_{x}^{m e s}-m_{y}^{m e s} \cdot \dot{m}_{x}^{e s t}}{\left(m_{x}^{m e s}\right)^{2}+\left(m_{y}^{m e s}\right)^{2}}
$$

where $\quad \dot{m}_{x}^{e s t}, \dot{m}_{y}^{e s t}$ and $\dot{m}_{z}^{\text {est }}$ are the three estimated magnetometer derivatives $\dot{m}_{x}^{e s t}, \dot{m}_{y}^{e s t}, \dot{m}_{z}^{e s t}$.

\subsection{Development of an Integrated Heading Solution}

The estimated angular rate of changes derived from magnetometer measurements $\left(\omega_{z}^{e s t}\right)$ is integrated with the measured angular rate of change collected from gyro sensor $\left(\omega_{z}^{m e s}\right)$ to provide heading using Kalman filter. The measurement equations can be written as:

$$
\begin{aligned}
& \psi_{k}^{e s t}=\psi_{k}+n_{k}^{e s t} \\
& \psi_{k}^{m e s}=\psi_{k}+b_{k}^{\psi}+n_{k}^{m e s}
\end{aligned}
$$

where

$$
b_{k+1}^{\psi}=b_{k}^{\psi}+T_{s} \cdot \dot{b}_{k+1}^{\psi},
$$

$T_{S}$ is the sampling time,

$\psi_{k}$ is the true heading at time $\mathrm{k}$,

$\psi_{k}^{e s t}$ is the heading based on the estimated angular rate of changes $\left(\omega_{z}^{e s t}\right)$ derived from magnetometer measurements,

$n_{k}^{e s t}$ is the measurement noise associated with magnetometer-based heading,

$\psi_{k}^{m e s}$ is the heading based on the measured angular rate of change collected from gyro sensor $\left(\omega_{z}^{m e s}\right)$,

$n_{k}^{m e s}$ is the measurement noise associated with gyro-based heading, $b_{k}^{\psi}$ is the bias associated with gyro-based heading and $\dot{b}_{k}^{\psi}$ is the derivative of the bias parameter that can be considered as it follows Gauss-Markov process (Gelb, 1974).

If the state space vector at time $\mathrm{k}$ is considered as:

$$
X_{k / k}=\left[\begin{array}{lll}
\psi_{k} & b_{k}^{\psi} & \dot{b}_{k}^{\psi}
\end{array}\right]
$$

Then, the integrated heading observation model in a compact form can be considered as:

$$
Z_{k}=\left[\begin{array}{l}
\psi_{z}^{e s t} \\
\psi_{z}^{\text {mes }}
\end{array}\right]=\left[\begin{array}{ccc}
1 & 0 & 0 \\
1 & 1 & 0
\end{array}\right] X_{k}
$$


And the integrated heading prediction model in a compact form can be considered as:

$$
X_{k+1}=\left\lfloor\begin{array}{ccc}
1 & 0 & 0 \\
0 & 1 & T_{s} \\
0 & 0 & e^{-\beta_{\psi} T_{s}}
\end{array}\right\rfloor X_{k}
$$

where $\beta_{\psi}$ is the Gauss-Markov model constant. The above prediction and observation models can be used to provide accurate integrated heading solution with Kalman filter estimator using MEMS-based gyro and magnetometer integrated system.

\section{RESULTS AND DISCUSSION}

To test the performance of the estimated heading, two data sets were implemented by the proposed integrated method. The first data set was conducted in static mode and without disturbances. The second data set was conducted in kinematic mode along horizontal straight line trajectory and with disturbances. The two data sets were collected with MEMSbased Xsens Inertial Measurement Unit (IMU) that composed of three accelerometers, three gyros and three magnetometers. Figure 1 and Table 1 show the Xsens IMU unit and characteristics.

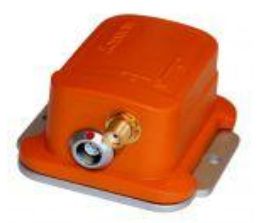

Figure 1. Xsens IMU (taken from taken from www.xsens.com)

\begin{tabular}{|c|c|c|c|c|}
\hline & & $\begin{array}{c}\text { rate of } \\
\text { turn }\end{array}$ & acceleration & $\begin{array}{l}\text { magnetic } \\
\text { field }\end{array}$ \\
\hline Unit & & {$[\mathrm{deg} / \mathrm{s}]$} & {$\left[\mathrm{m} / \mathrm{s}^{2}\right]$} & [mGauss] \\
\hline Dimensions & & 3 axes & 3 axes & 3 axes \\
\hline Full Scale & (units) & $+/-300$ & $+/-17$ & $+/-750$ \\
\hline Linearity & (\% of FS) & 0.1 & 0.2 & 0.2 \\
\hline Bias stability & (units $1 \sigma$ ) & 5 & 0.02 & 0.5 \\
\hline $\begin{array}{l}\text { Scale factor } \\
\text { stability }\end{array}$ & $(\% 1 \sigma)$ & - & 0.05 & 0.5 \\
\hline Noise density & (units $\sqrt{H z}$ ) & 0.1 & 0.001 & $0.5(1 \sigma)$ \\
\hline $\begin{array}{l}\text { Alignment } \\
\text { error }\end{array}$ & (deg) & 0.1 & 0.1 & 0.1 \\
\hline Bandwidth & $(\mathrm{Hz})$ & 40 & 30 & 10 \\
\hline
\end{tabular}

Table 1. Xsens IMU characteristics

Figure 2 shows the magnetometer-based heading solution, the gyro-based heading solution and the developed integrated heading solution with a static data set (without magnetic disturbances). The developed integrated heading solution shows superior results to the magnetometer-based and gyrobased heading estimations where magnetometer-based heading solution is very noisy and the gyro-based heading estimation largely drifts over time.

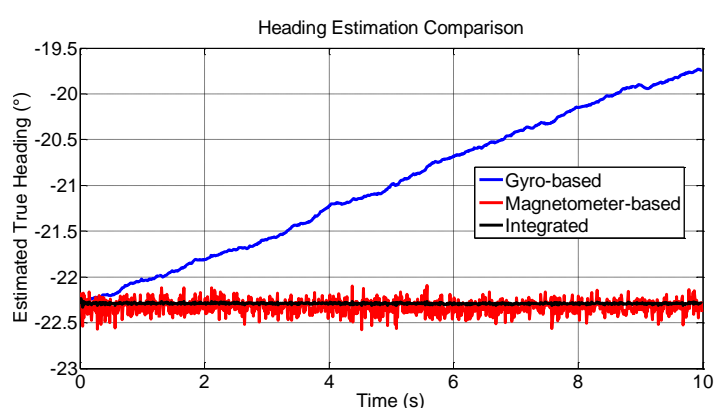

Figure 2. Heading estimation comparison between the gyrobased (blue), the magnetometer-based (red) and the developed integrated solutions (static case without magnetic disturbances)

Figure 3 shows the magnetometer-based heading solution, the gyro-based heading solution and the developed integrated heading solution with kinematic data set (with magnetic disturbances). The developed integrated heading solution shows superior results to magnetometer-based and gyro-based heading solutions where the magnetometer-based heading solution is severely disturbed and the gyro-based heading estimation drifts over time.

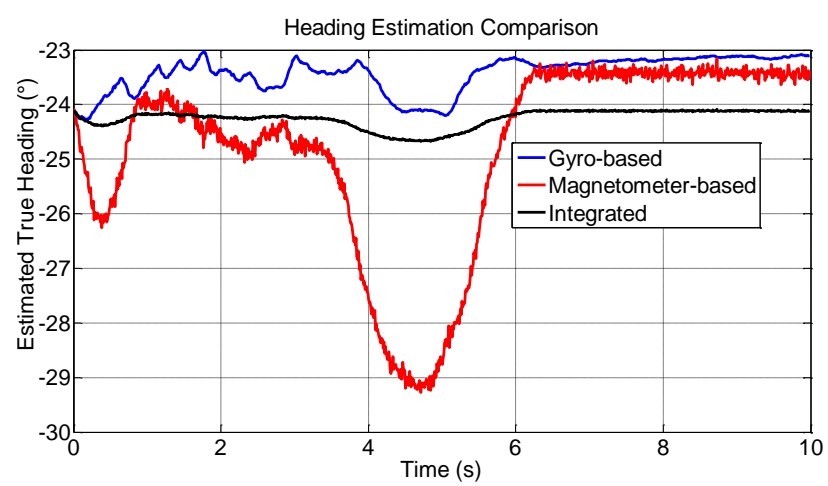

Figure 3. Heading solutions comparison between the gyrobased (blue), the magnetometer-based (red) and the proposed integrated solutions (kinematic case with magnetic disturbances)

The above results showed that the developed integrated heading estimation procedure provides accurate, smoothed and undisturbed solution when compared with magnetometer-based and gyro-based heading solutions.

\section{CONCLUSION}

This paper developed an accurate heading estimation procedure based on the integration of MEMS-based gyro and magnetometer measurements that correct gyro and magnetometer measurements where gyro angular rates of changes are estimated using magnetometer measurements and then integrated with the measured gyro angular rates of changes with a robust filter to estimate the heading. The proposed integrated solution is implemented by two data sets collected in static and kinematic modes and also collected without and with magnetic disturbances. The results showed that the developed integrated heading solution provides accurate, smoothed and undisturbed solution and superior to the magnetometer-based and gyro-based heading solutions. 


\section{REFERENCES}

Afzal, M.H., V. Renaudin, G.Lachapelle. (2011) Use of Earth's Magnetic Field for Mitigating Gyroscope Errors Regardless of Magnetic Perturbation. Sensors, 2011, 11, $11390-11414$.

Ali, A. S Siddharth, Z Syed, N El-Sheimy, 2012. An improved personal dead-reckoning algorithm for dynamically changing smartphone user modes, ION GNSS 2012, (Nashville), pp. 2432-2439.

El-Diasty, M., S. Pagiatakis, 2008. Calibration and Stochastic Modelling of Inertial Navigation Sensor Erros. In: Journal of Global Positioning System, Vol.7, No.2, pp. 170-182.

Gelb, A., 1974. “Applied Optimal Estimation.” The M.I.T. Press, Cambridge, Massachusetts.

Godha, S, G. Lachapelle, 2006. M.E. Canon, 2006. Integrated GPS/INS System for Pedestrian navigation in a Signal Degraded Environment ., ION GNSS 2006

Kubrak, K., 2007. Hybridisation of a GPS Receiver with LowCost Sensors for Personal Positioning in Urban Environment., Ph.D. Thesis, ENST.

Tian, Z., Y. Zhang, M. Zhou, Y. Liu, 2014. Pedestrian dead reckoning for MARG navigation using a smartphone. EURASIP Journal on Advances in Signal Processing.

Titterton, D. H., 2004. Strapdown inertial navigation technology. 2nd ed., Radar, sonar, navigations \& avionics. 